\title{
Cardiac Surgery in Germany during 2014: A Report on Behalf of the German Society for Thoracic and Cardiovascular Surgery
}

\author{
Andreas Beckmann ${ }^{1}$ Anne-Kathrin Funkat ${ }^{2}$ Jana Lewandowski ${ }^{1}$ Michael Frie ${ }^{3}$ Markus Ernst ${ }^{4}$ \\ Khosro Hekmat $^{5}$ Wolfgang Schiller ${ }^{6}$ Jan F. Gummert ${ }^{7}$ Joachim Thomas Cremer ${ }^{4}$
}

${ }^{1}$ German Society for Thoracic and Cardiovascular Surgery, Langenbeck-Virchow-Haus, Berlin, Germany

${ }^{2}$ Clinic for Cardiac Surgery, Heart Center Leipzig, University of Leipzig, Germany

${ }^{3}$ FOM Hochschule fuer Oekonomie \& Management, Essen, Germany

${ }^{4}$ Clinic for Cardiac and Vascular Surgery, University Kiel, Kiel, Germany

${ }^{5}$ Department of Cardiothoracic Surgery, University of Cologne, Köln, Germany

${ }^{6}$ Clinic for Cardiac Surgery, University of Bonn, Bonn, Germany

${ }^{7}$ Clinic for Thoracic and Cardiovascular Surgery, Heart and Diabetes

Center NRW, Bad Oeynhausen, Germany

Thorac Cardiovasc Surg 2015;63:258-269.
Address for correspondence Dr. med. Andreas Beckmann, Deutsche Gesellschaft für Thorax-, Herz- und Gefäßchirurgie [DGTHG], Langenbeck-Virchow-Haus, Luisenstr. 58-59, 10117 Berlin, Germany (e-mail: gf@dgthg.de).
Based on a voluntary registry of the German Society for Thoracic and Cardiovascular Surgery (GSTCVS), data of all heart surgery procedures performed in 78 German cardiac surgical units during the year 2014 are presented. In 2014, a total of 100,398 cardiac surgical procedures (implantable cardioverter-defibrillator and pacemaker procedures excluded) were submitted to the registry. More than $14.2 \%$ of the patients were older than 80 years, describing an increase of $0.4 \%$ compared with the previous year. The unadjusted in-hospital mortality for 40,006 isolated coronary artery bypass grafting procedures ( $84.7 \%$ on-pump, $15.3 \%$ off-pump) was $2.6 \%$. In 31,359 isolated valve procedures (including 9,194 catheter-based procedures), an in-hospital mortality of $4.4 \%$ was observed. This annual updated registry of the GSTCVS is published since 1989. It is an important tool for quality assurance and voluntary public reporting by illustrating current standards and actual developments for nearly all cardiac surgical procedures in Germany.

\section{Introduction}

Legitimate demands for a sophisticated quality assurance in medicine-by patients, relatives, insurance companies, and authorities all over the world-have stimulated the development of a wide range of registries and other activities to answer those needs. As early as 1978, the board of directors of the German Society for Thoracic and Cardiovascular Surgery (GSTCVS; www. dgthg.de) decided to set up an annually updated database of all cardiac surgical procedures in terms of a voluntary registry. Since 1989 , the annually updated data of the registry are published in the scientific journal of the GSTCVS. ${ }^{1-25}$ The aims of this registry

received

March 24, 2015

accepted

March 27, 2015

published online

May 26, 2015

are to gather developments and current trends in cardiac surgery in Germany, compiling various results for nearly all cardiac surgical procedures, enabling each participating cardiac surgical unit a comparison of its own results to the nationwide achievements, and enabling an evaluation on an international level for the society.

For monitoring actual conditions as well as the development in cardiac medicine, the registry covers all relevant techniques and also innovative technologies including offpump cardiac surgery, minimally invasive heart valve operations, and transcatheter aortic valve implantations [TAVI]. Thereby important findings for current patient

(c) 2015 Georg Thieme Verlag KG Stuttgart · New York
DOI http://dx.doi.org/ 10.1055/s-0035-1551676. ISSN 0171-6425. 
safety and the future of patient care may be collected and evaluated.

Data and results presented in this report comprehend assorted data of the year 2014.

\section{Materials and Methods}

Since 2004, a standardized questionnaire gathers detailed information about each individual procedure exactly defined by an annually updated German adaption of the International Classification of Procedures in Medicine (ICPM) called operation code (OPS-Operationen- und Prozedurenschlüssel).

All participants were requested to complete the structured questionnaire until January 16, 2015, asking for all performed procedures and associated in-hospital mortality. The recommended path for data export is an electronic transmission of an encrypted file which has to be addressed to the office of the GSTCVS in Berlin. After transaction, the data are decrypted, evaluated for completeness, and compiled for further analysis, thus ensuring anonymity for each participating institution. This compilation algorithm guarantees a high compliance for submission of complete datasets.

Inclusion criteria for the registry 2014 were all patients receiving cardiac surgical procedures performed between January 1, 2014, and December 31, 2014, unrelated to the date of admission or discharge as compared with other registries. Alike to all previous years, the number of procedures was counted rather than individual patients. For example, if a patient required additional coronary bypass grafting due to a complication after initial aortic valve replacement during one admission, one count in the category "aortic valve replacement" and another in the category "coronary surgery" are enumerated. Thus, the registry contains more procedures than the real number of operated patients.

Death of patients was defined as in-hospital mortality. Per definition, the observed mortality is always attributed to the first cardiac procedure; for example, the death of a patient requiring coronary bypass grafting due to a complication of an aortic valve procedure would only be attributed to the aortic valve procedure.

The main reason for this structural setup of the registryestablished over several decades-is to keep in accordance with the German data privacy act with its specific regulations for patients. Furthermore, it seemed to be relevant to get detailed information about all performed procedures and not only the number of treated patients. Last but not least, the process of data acquisition had to be standardized and feasible for all cardiac surgery units in Germany, thus enabling the submission of a complete dataset, regardless of the locally existing hard- and software used for data management.

In 2014, a total of 78 institutions performed heart surgery. Fortunately all units answered the questionnaire and delivered a complete dataset for the year 2014 including hospital mortality rates.

\section{Registry Data 2014}

-Table 1 illustrates the development of procedures using extracorporeal circulation (ECC) in Germany over one decade.
Since 2010, the number of heart operations using ECC shows only a slight decline.

Overall 187,392 procedures were reported to the registry for the year 2014, an increase of 3.9\% (2013: 180,367 procedures). A total of 104,484 cardiac surgical procedures (excluded: ICD, pacemakers and miscellaneous procedures without ECC) reveal an increase of $1.6 \%(n=1,639)$ compared with the year 2013 (102,845 procedures) (- Table 2). - Tables 3 to 5, V1 to V7, C1 to C2, C3, - Table Con1 and Con2, and - Table Mis 1 to 5 and -Figs. 1-9 demonstrate the compiled registry data of 2014 for various categories.

Compared to the data of previous years, several important developments continued in 2014 almost unchanged. Over the past 10 years, the age distribution of patients ( - Fig. $\mathbf{6}$ ) showed a shift to elderly patients with presently $54.3 \%$ of the cardiac procedures performed in patients of at least 70 years of age and $14.2 \%$ in patients of 80 years or older. However, mortality remained on the same low level or even decreased slightly over the last decade (-Fig. $\mathbf{2}$ ). The rate of coronary artery bypass grafting $(\mathrm{CABG})$ procedures decreased over the past years while the relative number of off-pump CABG showed no relevant variation (2014: 15.3\%, 2013: 15.5\%) (-Fig. 3).

Since 2004 , more than $50 \%$ of isolated mitral valve procedures were reconstructions. In 2014, the mitral valve reconstruction rate was $65.7 \%$ ( - Fig. 8). Based on the fact that all isolated mitral valve procedures are included in this registry, these data have to be interpreted with caution. Regardless of the underlying valve disease/morphology or urgency of operation, it has to be assumed that the relative rate of mitral valve reconstruction would certainly be even higher if patients without possibility or indication for reconstruction would have been excluded (e.g., mitral valve stenosis, calcifications, or endocarditis). In other publications, ${ }^{26}$ patients with mitral valve stenosis, endocarditis, and emergency procedures were excluded.

The continued increase of left ventricular assist device implantations (-Fig. 10) emphasizes the increasing relevance of mechanical circulatory support.

Again a remarkable trend is the still ongoing increase of TAVI procedures in Germany (-Fig. 5), while the number of isolated aortic valve replacement procedures remained on a nearly unchanged level. Starting in 2006 with just 78 implantations ( $0.67 \%$ of isolated aortic valve procedures), in 2014 a total of 8,631 (42.3\%) TAVIs were reported to the registry. It must be emphasized that the 78 institutions which contribute their data to this registry do not represent all departments performing TAVI in Germany. It is well known that some additional institutions in Germany perform TAVI procedures under different infrastructure conditions and various external cooperations. This practice does not correlate to the recommendations of the European guideline ${ }^{27}$ on the management of valvular heart disease. Considering this background, on January 22, 2015, the Federal Joint Committee (G-BA)-as the highest decision-making body of the joint self-government in Germany-issued the first version of the quality assurance directive for "minimal invasive heart valve interventions (TAVI, Mitral Clip reconstruction)" in which obligatory structures, defined processes, and qualified personnel are exactly specified. ${ }^{28}$ 
In addition, the short-, mid-, and long-term results of the German Aortic Valve Registry (GARY) ${ }^{29-33}$ and the annual analyses of the legal quality assurance ( $\$ 137$ SGB V) are of outstanding patient benefit.

\section{Discussion}

The registry of the GSTCVS enables a comprehensive overview of all cardiac surgical procedures performed in Germany during 2014. The accuracy of this registry is considered to be high due to the implemented compilation algorithm using standardized operation coding as a relevant criterion for reimbursement purposes. This is supported by other authors who could demonstrate a high accuracy for major outcome parameters in unaudited registries. ${ }^{30}$ In continuation with previous years, it can be concluded that cardiac surgery in Germany is performed on a constantly high level with high in-hospital survival rates compared with other international registries. This conclusion is especially important in an era of demographic change of the German population, resulting in a continuous increase of patient age and related comorbidities, both leading to a higher preoperative risk profile.
Compared with 2013, the count of cardiac surgery procedures remains almost on the same level, which is partly due to the still increasing number of catheter-based heart valve procedures.

Further improvements in the basic configuration of the registry are recommended to enable a more detailed and particularly a risk-adjusted data analyses. ${ }^{34}$ However, if significant structural changes of data collection for the registry are conducted, it must be ensured that data compatibility still allows further longitudinal data analysis.

Completeness, validity, and further developments will depend on continued efforts of the GSTCVS in close collaboration with all cardiac surgical units in Germany. This will be of outstanding importance in the sense of a contribution for patient safety and to adduce evidence for the quality of cardiac surgery in Germany.

\section{Acknowledgments}

On behalf of the German Society for Thoracic and Cardiovascular Surgery, the authors would like to thank the heads of departments of all cardiac surgery units in Germany and their employees for their continued cooperation and support to realize this registry.

\section{Appendix: Tables and Figures}

Table 1 Open heart procedures using extracorporeal circulation (2005-2014)

\begin{tabular}{|l|l|l|l|l|l|l|l|l|l|l|}
\hline & 2005 & 2006 & 2007 & 2008 & 2009 & 2010 & 2011 & 2012 & 2013 & 2014 \\
\hline Units & 79 & 80 & 80 & 79 & 80 & 79 & 78 & 79 & 79 & 78 \\
\hline Operations & 91,967 & 91,057 & 91,618 & 89,773 & 86,916 & 84,686 & 84,402 & 84,388 & 84,040 & 83,787 \\
\hline Average per unit & 1,164 & 1,138 & 1,145 & 1,136 & 1,086 & 1,072 & 1,082 & 1,068 & 1,064 & 1,074 \\
\hline
\end{tabular}

Table 2 Cardiac surgery categories (comparison: 2014 vs. 2013)

\begin{tabular}{|l|l|l|l|l|}
\hline Category & $\begin{array}{l}\text { With } \\
\text { ECC }\end{array}$ & $\begin{array}{l}\text { Without } \\
\text { ECC }\end{array}$ & Total change \\
\hline Heart valve procedures & 22,335 & 9,024 & 31,359 & +5.7 \\
\hline Coronary artery bypass grafting & 47,246 & 6,559 & 53,805 & -0.8 \\
\hline Surgery for congenital heart disease & 4,755 & 1,024 & 5,779 & 7,755 \\
\hline Surgery of thoracic aorta & 7,117 & 638 & 2,474 & +3.6 \\
\hline Cardiac surgery, other & 1,172 & 1,302 & 2,910 & -5.5 \\
\hline Assist device procedures & 760 & 2,150 & 26,252 & +9.5 \\
\hline Pacemaker and ICD procedures & 40 & 26,212 & 57,058 & +2.0 \\
\hline Extracardiac surgery & 362 & 56,696 & 187,392 & +9.4 \\
\hline Total & 83,787 & 103,605 & & +3.9 \\
\hline
\end{tabular}

Abbreviation: ECC, extracorporeal circulation.

Table 3 Units assorted by volume categories (procedures with or without ECC)

\begin{tabular}{|l|l|l|l|l|l|}
\hline Quantity of operations & $<\mathbf{5 0 0}$ & $\mathbf{5 0 0 - 9 9 9}$ & $\mathbf{1 , 0 0 0 - 1 , 4 9 9}$ & $\mathbf{1 , 5 0 0 - 1 , 9 9 9}$ & $\mathbf{2 , 0 0 0 - 5 , 0 0 0}$ \\
\hline Number of units & 7 & 20 & 26 & 12 & 13 \\
\hline Average per unit & 342 & 928 & 1,152 & 1,864 & 2,087 \\
\hline Min-Max & $238-427$ & $546-988$ & $1,027-1,495$ & $1,513-1,992$ & $2,045-3,972$ \\
\hline
\end{tabular}

Abbreviation: ECC, extracorporeal circulation. 
Cardiac Surgery in Germany during 2014 Beckmann et al. 261

Table 4 Total number of units according to surgical procedures in 2014

\begin{tabular}{|l|l|}
\hline Type of surgery performed & $\begin{array}{l}\text { Number } \\
\text { of units }\end{array}$ \\
\hline Coronary artery bypass grafting & 77 \\
\hline Heart valve surgery & 77 \\
\hline Surgery for CHD in children $<1$ y with ECC & $23^{\mathrm{a}}$ \\
\hline Heart transplantation & $21^{\mathrm{b}}$ \\
\hline Heart-lung transplantation & 5 \\
\hline
\end{tabular}

Abbreviations: CHD, congenital heart disease; ECC, extracorporeal circulation.

${ }^{a} n=2,090$ thereof: $7-19$ procedures in 4 units, $23-47$ procedures in 4 units, 59-90 procedures in 7 units, 104-296 procedures in 8 units.

${ }^{b} n=294: 58 \%$ of heart transplantations (HTx) in 2014 were performed by 6 of 21 units with $\geq 15$ HTx per year; thereof: 1-3 HTx in 4 units, 5-9 HTx in 5 units, 12-18 HTx in 10 units, 21-85 HTx in 2 units.

Table V1 Isolated valve procedures (categories)

\begin{tabular}{|l|l|l|l|}
\hline Procedures & N & Deaths & \% \\
\hline Single & 18,408 & 625 & 3.4 \\
\hline Double & 3,288 & 314 & 9.5 \\
\hline Triple & 372 & 50 & 13.4 \\
\hline $\begin{array}{l}\text { Transcatheter access } \\
\text { (single value) }\end{array}$ & 9,184 & 386 & 4.2 \\
\hline $\begin{array}{l}\text { Transcatheter access } \\
\text { (double value) }\end{array}$ & 10 & 1 & 10.0 \\
\hline Not specified & 97 & 8 & 8.2 \\
\hline Total & 31,359 & 998 & 3.2 \\
\hline
\end{tabular}

Notes: Combined procedures (with coronary artery bypass grafting, aortic surgery) are excluded.

Transcatheter valve procedures: 8,631 aortic valve implantations; 91 mitral valve implantations; 457 mitral valve repairs; 3 tricuspid valve implantations; 2 tricuspidal valve repairs; 10 combined procedures, aortic and mitral valve procedure; no pulmonary valve implantation.

Table V3 Isolated aortic valve procedures (prosthesis)

\begin{tabular}{|l|l|l|l|}
\hline Prosthesis/native valve & $\boldsymbol{N}$ & Deaths & $\%$ \\
\hline Mechanical prosthesis & 1,360 & 29 & 2.1 \\
\hline Xenograft & 10,375 & 292 & 2.8 \\
\hline Homograft & 29 & 2 & 6.9 \\
\hline Reconstruction & 117 & 2 & 1.7 \\
\hline Total & 11,881 & 325 & 2.7 \\
\hline
\end{tabular}

Notes: A total of 2,781 (23.4\%) procedures were performed by a partial sternotomy. Transcatheter procedures and apical aortic conduits procedures $(n=1)$ are not included.
Table 5 Additional data for cardiac procedures with ECC in 2014 and 2013

\begin{tabular}{|l|l|l|l|l|}
\hline & \multicolumn{2}{|l|}{2014} & 2013 \\
\hline Emergency operations & 12,583 & $12.0 \%$ & 11,944 & $11.6 \%$ \\
\hline Redo procedures & 8,809 & $8.4 \%$ & 8,284 & $8.1 \%$ \\
\hline
\end{tabular}

Note: The numbers in each category reflect procedures and not individual patients.

Table V2 Single valve procedures (access type)

\begin{tabular}{|l|l|l|l|}
\hline Heart valve/access & $N$ & Deaths & $\%$ \\
\hline Aortic valve & 9,100 & 282 & 3.1 \\
\hline Sternotomy & 2,781 & 43 & 1.5 \\
\hline Partial sternotomy & 5,570 & 184 & 3.3 \\
\hline Transvascular & 3,061 & 167 & 5.5 \\
\hline Transapical & \multicolumn{5}{l|}{} \\
\hline Mitral valve & 3,121 & 200 & 6.4 \\
\hline Sternotomy & 2,792 & 43 & 1.5 \\
\hline Minimal invasive & 548 & 33 & 6.0 \\
\hline Transcatheter & \multicolumn{5}{|l}{} \\
\hline Tricuspid valve & 428 & 50 & 11.7 \\
\hline Sternotomy & 133 & 6 & 4.5 \\
\hline Minimal invasive & 5 & 2 & 40.0 \\
\hline Transcatheter & \multicolumn{5}{|l}{} \\
\hline Pulmonary valve & 50 & 1 & 2.0 \\
\hline Sternotomy & 2 & 0 & 0.0 \\
\hline Minimal invasive & 0 & 0 & - \\
\hline Transcatheter & 27,591 & 1,011 & 3.7 \\
\hline Total & \multicolumn{5}{|l}{} \\
\hline
\end{tabular}

Notes: The absolute quantity of isolated aortic valve procedures via sternotomy remained on a constant level counting 11,881 in 2014 compared with 11,891 procedures in 2013.

A total of 2,792 (47.2\%) mitral valve procedures were performed by a minimally invasive access.

Transcatheter procedures and apical aortic conduits procedures $(n=1)$ are not included.

Table V4 Isolated mitral valve procedures (prosthesis)

\begin{tabular}{|l|l|l|l|}
\hline Prosthesis/native valve & $N$ & Deaths & $\%$ \\
\hline Mechanical prosthesis & 488 & 34 & 7.0 \\
\hline Xenograft & 1,530 & 145 & 9.5 \\
\hline Homograft & 8 & 1 & 12.5 \\
\hline Reconstruction & 3,887 & 63 & 1.6 \\
\hline Total & 5,913 & 243 & 4.1 \\
\hline
\end{tabular}

Notes: A total of 2,792 (47.2\%) were performed via minimally invasive access. Transcatheter procedures are excluded. 
262 Cardiac Surgery in Germany during 2014 Beckmann et al.

Table V5 Multiple valve procedures (categories)

\begin{tabular}{|l|l|l|l|}
\hline Combination & N & Deaths & $\%$ \\
\hline Aortic + mitral & 1,335 & 144 & 10.8 \\
\hline Mitral + tricuspid & 1,640 & 145 & 8.8 \\
\hline Aortic + tricuspid & 220 & 22 & 10.0 \\
\hline Tricuspid + pulmonary & 11 & 2 & 18.2 \\
\hline Aortic + pulmonary & 82 & 1 & 1.2 \\
\hline Aortic + mitral + tricuspid & 369 & 49 & 13.3 \\
\hline Aortic + mitral + pulmonary & 3 & 1 & 33.3 \\
\hline Total & 3,660 & 364 & 9.9 \\
\hline
\end{tabular}

Note: Transcatheter procedures are excluded.

ancluding Ross procedures.

Table V6 Mitral valve surgery-implantation/replacement versus repair

\begin{tabular}{|c|c|c|c|c|c|c|c|c|c|c|}
\hline \multirow[t]{2}{*}{ Mitral valve surgery } & \multirow[t]{2}{*}{$N$} & \multirow{2}{*}{$\begin{array}{l}\text { Total } \\
\text { deaths }\end{array}$} & \multirow{2}{*}{$\begin{array}{l}\% \\
\text { death }\end{array}$} & \multirow[t]{2}{*}{ \% repair } & \multicolumn{3}{|c|}{ Implantation/replacement } & \multicolumn{3}{|l|}{ Repair } \\
\hline & & & & & $N$ & Deaths & $\%$ death & $N$ & Deaths & $\%$ death \\
\hline Isolated & 5,913 & 243 & 4.1 & 65.7 & 2,026 & 180 & 8.9 & 3,887 & 63 & 1.6 \\
\hline $\begin{array}{l}\text { Mitral valve }+ \\
\text { aortic valve }\end{array}$ & 1,335 & 144 & 10.8 & 47.0 & 707 & 104 & 14.7 & 628 & 0 & 0.0 \\
\hline Tricuspid valve repair $^{\mathrm{a}}$ & 1,602 & 137 & 8.6 & 65.5 & 552 & 1 & 0.2 & 1,050 & 81 & 7.7 \\
\hline CABG & 2,630 & 256 & 9.7 & 70.1 & 787 & 124 & 15.8 & 1,843 & 132 & 7.2 \\
\hline $\begin{array}{l}\text { CABG + aortic } \\
\text { valve replacement }\end{array}$ & 658 & 113 & 17.2 & 55.9 & 290 & 58 & 20.0 & 368 & 55 & 14.9 \\
\hline Total & 12,138 & 893 & 7.4 & 64.1 & 4,362 & 467 & 10.7 & 7,776 & 331 & 4.3 \\
\hline
\end{tabular}

${ }^{a}$ Thirty-eight procedures (not specified mitral valve + tricuspid valve surgery) are excluded. Deaths: 21.1 (8/38).

Table V7 Transcatheter heart valve procedures

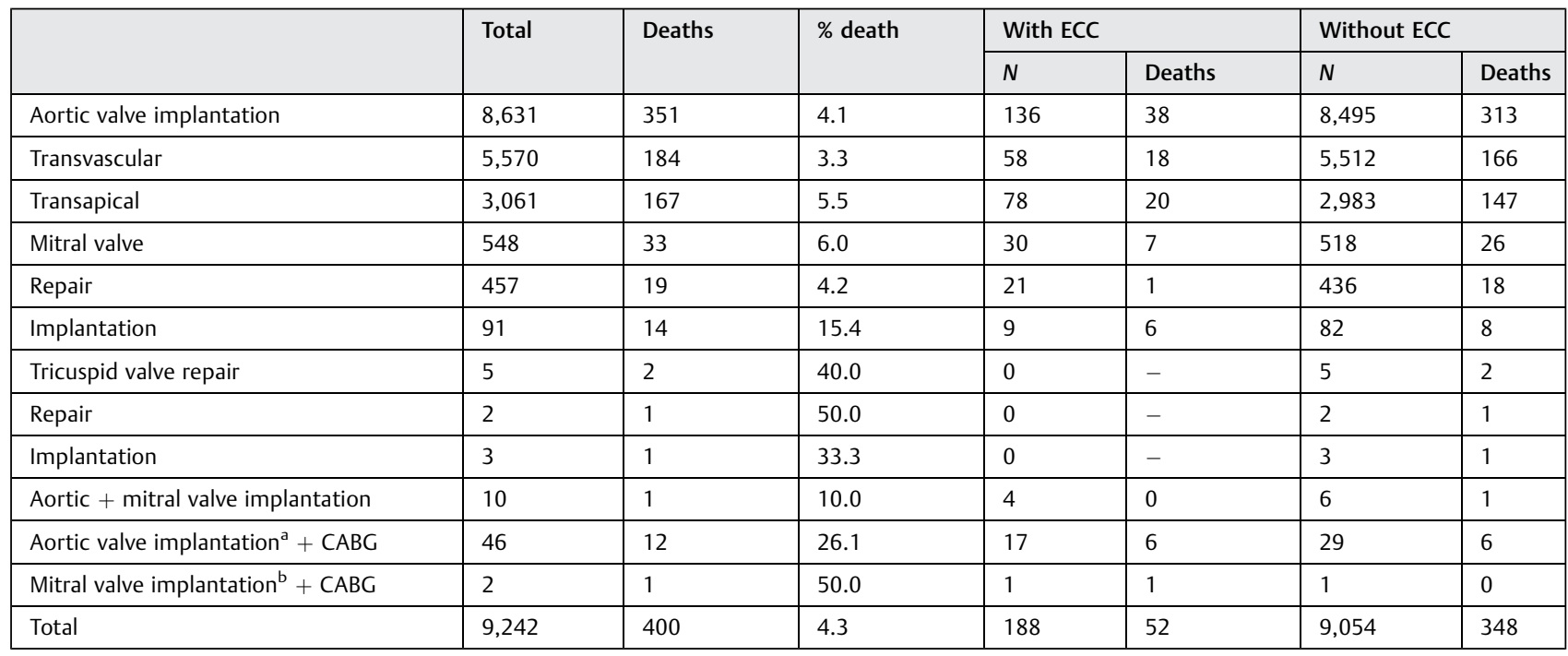

Abbreviations: CABG, coronary artery bypass grafting; ECC, extracorporeal circulation.

Notes: Pulmonary valve implantations for the correction of congenital heart defects are not included; no procedure for adults without congenital lesion was reported; $35.5 \%$ of transcatheter aortic valve implantations (TAVIs) were performed by transapical access; $1.6 \%$ of TAVI procedures were performed using ECC. It has to be assumed that use of ECC is mostly an emergent procedure in a life-threatening situation for the patient resulting in an observed mortality of $27.9 \%$ in this group. Nevertheless, this underlines the necessity of well-defined directives including infrastructure, processes, and qualified employees for invasive heart valve procedures (e.g., TAVI).

${ }^{\mathrm{a}}$ Femoral, subclavian, or transaortic access.

${ }^{\mathrm{b}}$ Transvascular and transapical access. 
Table Con1 Congenital heart surgery with/without ECC

\begin{tabular}{|c|c|c|c|}
\hline Age (y) & $N$ & Deaths & $\%$ \\
\hline \multicolumn{4}{|c|}{ Without ECC } \\
\hline$\geq 18$ & 37 & 0 & 0.0 \\
\hline $1-17$ & 195 & 0 & 0.0 \\
\hline$<1$ & 792 & 24 & 3.0 \\
\hline Total & 1024 & 24 & 2.3 \\
\hline \multicolumn{4}{|l|}{ With ECC } \\
\hline$\geq 18$ & 934 & 26 & 2.8 \\
\hline 1 to 17 & 1731 & 11 & 0.6 \\
\hline$<1$ & 2090 & 59 & 2.8 \\
\hline Total & 4755 & 96 & 2.0 \\
\hline
\end{tabular}

Abbreviation: ECC, extracorporeal circulation.

Table Con2 Procedures for congenital heart disease with and without ECC

\begin{tabular}{|c|c|c|c|c|c|c|c|c|c|}
\hline \multirow[t]{2}{*}{ Lesion } & \multicolumn{3}{|c|}{ Age $<1$ y } & \multicolumn{3}{|c|}{ Age $1-17 y$} & \multicolumn{3}{|c|}{ Age $\geq 18$ y } \\
\hline & $N$ & Deaths & $\%$ & $N$ & Deaths & $\%$ & $N$ & Deaths & $\%$ \\
\hline ASD & 52 & 1 & 1.9 & 263 & 0 & 0.0 & 266 & 4 & 1.5 \\
\hline Complete AV-canal & 193 & 6 & 3.1 & 77 & 2 & 2.6 & 24 & 1 & 4.2 \\
\hline VSD & 318 & 3 & 0.9 & 89 & 0 & 0.0 & 18 & 0 & 0.0 \\
\hline Fallot tetralogy & 202 & 2 & 1.0 & 30 & 1 & 3.3 & 1 & 0 & 0.0 \\
\hline DORV & 49 & 0 & 0.0 & 23 & 1 & 4.3 & 0 & - & - \\
\hline TGA & 142 & 2 & 1.4 & 6 & 0 & 0.0 & 0 & - & - \\
\hline TGA + VSD & 62 & 2 & 3.2 & 17 & 0 & 0.0 & 1 & 0 & 0.0 \\
\hline Truncus arteriosus & 42 & 2 & 4.8 & 5 & 0 & 0.0 & 0 & - & - \\
\hline Fontan & 1 & 0 & 0.0 & 232 & 1 & 0.4 & 6 & 0 & 0.0 \\
\hline Norwood & 160 & 19 & 11.9 & 0 & - & - & 0 & - & - \\
\hline Pulmonary valve & 58 & 1 & 1.7 & 249 & 0 & 0.0 & 86 & 1 & 1.2 \\
\hline Transcatheter pulmonary valve implantation & 0 & - & - & 7 & 0 & 0.0 & 14 & 0 & 0.0 \\
\hline Aortic valve & 59 & 0 & 0.0 & 215 & 0 & 0.0 & 304 & 9 & 3.0 \\
\hline Ross procedure & 10 & 3 & 30.0 & 27 & 0 & 0.0 & 13 & 1 & 7.7 \\
\hline Mitral valve & 48 & 0 & 0.0 & 111 & 1 & 0.9 & 88 & 6 & 6.8 \\
\hline Tricuspid valve & 88 & 1 & 1.1 & 70 & 0 & 0.0 & 42 & 4 & 9.5 \\
\hline PDA & 251 & 5 & 2.0 & 13 & 0 & 0.0 & 2 & 0 & 0.0 \\
\hline Coarctation & 202 & 1 & 0.5 & 35 & 0 & 0.0 & 9 & 0 & 0.0 \\
\hline Heart transplant & 4 & 0 & 0.0 & 25 & 0 & 0.0 & 0 & - & - \\
\hline Heart + lung transplant & 0 & - & - & 0 & - & - & 0 & - & - \\
\hline Lung transplant & 0 & - & - & 10 & - & 0.0 & 0 & - & - \\
\hline Others & 962 & 35 & 3.6 & 451 & 6 & 1.3 & 97 & 0 & 0.0 \\
\hline Total & 2903 & 83 & 2.9 & 1955 & 12 & 0.6 & 971 & 26 & 2.7 \\
\hline
\end{tabular}

Abbreviations: ASD, atrial septal defect; DORV, double outlet right ventricle; ECC, extracorporeal circulation; PDA, persistant ductus arteriosus; TGA, transposition of great arteries; VSD, ventricular septal defect. 
264 Cardiac Surgery in Germany during 2014 Beckmann et al.

Table C1 Isolated CABG and combined procedures with ECC

\begin{tabular}{|c|c|c|c|}
\hline Procedures & $N$ & Deaths & $\%$ \\
\hline CABG & 40,006 & 1,042 & 2.6 \\
\hline \multicolumn{4}{|l|}{$\mathrm{CABG}+$} \\
\hline $\begin{array}{l}\text { Aortic valve } \\
\text { replacement }\end{array}$ & 7,809 & 382 & 4.9 \\
\hline Mitral valve repair & 1,843 & 132 & 7.2 \\
\hline $\begin{array}{l}\text { Mitral valve } \\
\text { replacement }\end{array}$ & 787 & 124 & 15.8 \\
\hline $\begin{array}{l}\text { Aortic valve } \\
\text { replacement }+ \text { mi- } \\
\text { tral valve repair }\end{array}$ & 368 & 55 & 14.9 \\
\hline $\begin{array}{c}\text { Aortic }+ \text { mitral } \\
\text { valve replacement }\end{array}$ & 290 & 58 & 20.0 \\
\hline $\begin{array}{l}\text { Aneurysmal } \\
\text { resection }\end{array}$ & 152 & 5 & 3.3 \\
\hline $\begin{array}{l}\text { Transcatheter } \\
\text { aortic valve } \\
\text { implantation }\end{array}$ & 46 & 12 & 26.1 \\
\hline TMLR & 0 & - & - \\
\hline Other & 2,502 & 131 & 5.2 \\
\hline Total & 53,803 & 1,941 & 3.6 \\
\hline
\end{tabular}

Abbreviations: CABG, coronary artery bypass grafting; ECC, extracorporeal circulation; TMLR, transmyocardial laser revascularization.
Table C2 Isolated CABG with ECC

\begin{tabular}{|l|l|l|l|}
\hline Number of grafts & $N$ & Deaths & \% \\
\hline Single & 1,193 & 63 & 5.3 \\
\hline Double & 7,146 & 243 & 3.4 \\
\hline Triple & 15,018 & 401 & 2.7 \\
\hline Quadruple & 8,099 & 182 & 2.2 \\
\hline Quintuple + more & 2,422 & 54 & 2.2 \\
\hline Total & 33,878 & 943 & 2.8 \\
\hline
\end{tabular}

Abbreviations: CABG, coronary artery bypass grafting; ECC, extracorporeal circulation.

Table C3 Isolated CABG off-pump

\begin{tabular}{|l|l|l|l|}
\hline Number of grafts & $\boldsymbol{N}$ & Deaths & \% \\
\hline Single & 1,361 & 17 & 1.2 \\
\hline Double & 1,765 & 42 & 2.4 \\
\hline Triple & 2,092 & 29 & 1.4 \\
\hline Quadruple & 751 & 8 & 1.1 \\
\hline Quintuple + more & 159 & 3 & 1.9 \\
\hline Total & 6,128 & 99 & 1.6 \\
\hline
\end{tabular}

Abbreviation: CABG, coronary artery bypass grafting.

Table Mis1 Ross procedures (age groups)

\begin{tabular}{|c|c|c|c|c|c|c|c|c|c|c|}
\hline Autologous AVR and PVR & 2005 & 2006 & 2007 & 2008 & 2009 & 2010 & 2011 & 2012 & 2013 & 2014 \\
\hline In patients $\geq 18 \mathrm{y}$ & 235 & 228 & 261 & 207 & 175 & 184 & 134 & 117 & 107 & 90 \\
\hline In patients $<18 \mathrm{y}$ & 46 & 50 & 34 & 42 & 54 & 43 & 40 & 36 & 33 & 37 \\
\hline Total & 281 & 278 & 295 & 249 & 229 & 227 & 174 & 153 & 140 & 127 \\
\hline
\end{tabular}

Table Mis2 Heart and lung transplantation

\begin{tabular}{|l|l|l|l|l|l|l|}
\hline \multirow{2}{*}{} & \multicolumn{2}{|l|}{ With ECC } & \multicolumn{2}{l|}{ Without ECC } \\
\cline { 2 - 7 } & N & Deaths & $\%$ & N & Deaths \\
\hline HTx & 294 & 33 & 11.2 & & & \\
\hline HLTx & 9 & 4 & 44.4 & & & \\
\hline LTx & 62 & 7 & 11.3 & 244 & 13 \\
\hline
\end{tabular}

Abbreviation: ECC, extracorporeal circulation.

Notes: All pediatric transplantations (demonstrated in table Con3) are included in this table.

Eurotransplant (ET) has reported for the same period 292 heart transplantations (HTx), 3 heart + kidney transplantations, 0 heart + liver transplantations, 9 heart-lung transplantations (HLTx), 296 double lung (DLTx), 46 single lung transplantations (SLTx), 0 lung + kidney transplantations, and 1 lung + liver transplantations.

Table Mis3 Aortic surgery

\begin{tabular}{|c|c|c|c|c|c|c|}
\hline \multirow[t]{2}{*}{ Replacement $^{\mathrm{a}}$} & \multicolumn{3}{|c|}{ With ECC } & \multicolumn{3}{|c|}{ Without ECC } \\
\hline & $N$ & Deaths & $\%$ & $N$ & Deaths & $\%$ \\
\hline Supracoronary ascending aorta & 1,560 & 118 & 7.6 & & & \\
\hline Supracoronary ascending aorta + AVR & 1,218 & 47 & 3.9 & & & \\
\hline
\end{tabular}


Table Mis3 (Continued)

\begin{tabular}{|c|c|c|c|c|c|c|}
\hline \multirow[t]{2}{*}{ Replacement $^{a}$} & \multicolumn{3}{|c|}{ With ECC } & \multicolumn{3}{|c|}{ Without ECC } \\
\hline & $N$ & Deaths & $\%$ & $N$ & Deaths & $\%$ \\
\hline \multicolumn{7}{|l|}{ Infracoronary ascending aorta } \\
\hline Mechanical heart valve conduits & 456 & 22 & 4.8 & & & \\
\hline Biological heart valve conduits & 891 & 98 & 11.0 & & & \\
\hline David procedure & 493 & 7 & 1.4 & & & \\
\hline Yacoub procedure & 117 & 7 & 6.0 & & & \\
\hline Other & 267 & 15 & 5.6 & & & \\
\hline Aortic arch ${ }^{b}$ & 1,968 & 241 & 12.2 & & & \\
\hline Descending aorta & 68 & 14 & 20.6 & 6 & 1 & 16.7 \\
\hline Thoracoabdominal aorta & 76 & 10 & 13.2 & 18 & 7 & 38.9 \\
\hline Endostent descending aorta & 3 & 1 & 33.3 & 614 & 35 & 5.7 \\
\hline Total & 7,117 & 580 & 8.1 & 638 & 43 & 6.7 \\
\hline
\end{tabular}

Notes: All procedures involving aortic surgery are included in this table (Isolated and all possible combined procedures, e.g., additional coronary artery bypass grafting).

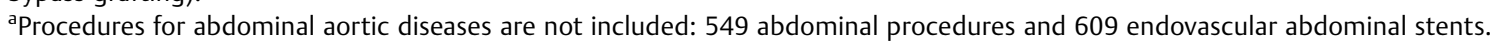

${ }^{b}$ All possible combined procedures are included in this category; the only common denominator is aortic arch surgery.

Table Mis4 Pacemaker and ICD procedures

\begin{tabular}{|c|c|c|c|c|c|c|c|}
\hline \multirow[t]{2}{*}{ Device/category } & \multirow[t]{2}{*}{$N$} & \multirow[t]{2}{*}{ Deaths } & \multirow[t]{2}{*}{ \% death } & \multicolumn{2}{|c|}{ With ECC } & \multicolumn{2}{|c|}{ Without ECC } \\
\hline & & & & $N$ & Deaths & $N$ & Deaths \\
\hline Pacemaker & 14,398 & 97 & 0.7 & 14 & 3 & 14,384 & 94 \\
\hline Implantation & 9,299 & 67 & 0.7 & 0 & 0 & 9,299 & 67 \\
\hline Battery exchange & 2,033 & 0 & 0.0 & 0 & 0 & 2,033 & 0 \\
\hline Revision & 3,066 & 30 & 1.0 & 14 & 3 & 3,052 & 27 \\
\hline$I C D$ & 10,104 & 73 & 0.7 & 25 & 4 & 10,079 & 69 \\
\hline Implantation & 4,660 & 17 & 0.4 & 0 & 0 & 4,660 & 17 \\
\hline Battery exchange & 2,031 & 4 & 0.2 & 0 & 0 & 2,031 & 4 \\
\hline Revision & 3,413 & 52 & 1.5 & 25 & 4 & 3,388 & 48 \\
\hline Miscellaneous & 1,750 & 10 & 0.6 & 1 & 1 & 1,749 & 9 \\
\hline Total & 26,252 & 180 & 0.7 & 40 & 8 & 26,212 & 172 \\
\hline
\end{tabular}

Table Mis5 Surgical ablation procedures for arrhythmia

\begin{tabular}{|l|l|l|l|}
\hline & Total & Endocardiac $(\boldsymbol{n})$ & Epicardiac $(\boldsymbol{n})$ \\
\hline Energy source & & & \\
\hline Unipolar radiofrequency & 205 & 92 & 113 \\
\hline Unipolar cryo-radiofrequency & 329 & 201 & 128 \\
\hline Bipolar radiofrequency & 2,396 & 312 & 2,084 \\
\hline Cryothermy & 1,760 & 1,464 & 296 \\
\hline Microwave & 17 & 4 & 13 \\
\hline Focused ultrasound & 224 & 28 & 196 \\
\hline Laser & 0 & 0 & 0 \\
\hline Other & 20 & 7 & 13 \\
\hline Total & 4,951 & 2,108 & 2,843 \\
\hline
\end{tabular}

Notes: All isolated ablation procedures and all possible combination of procedures (e.g., coronary artery bypass grafting + ablation) are included. A total of 484 procedures are not specified with regard to endocardiac/epicardiac ablation. 


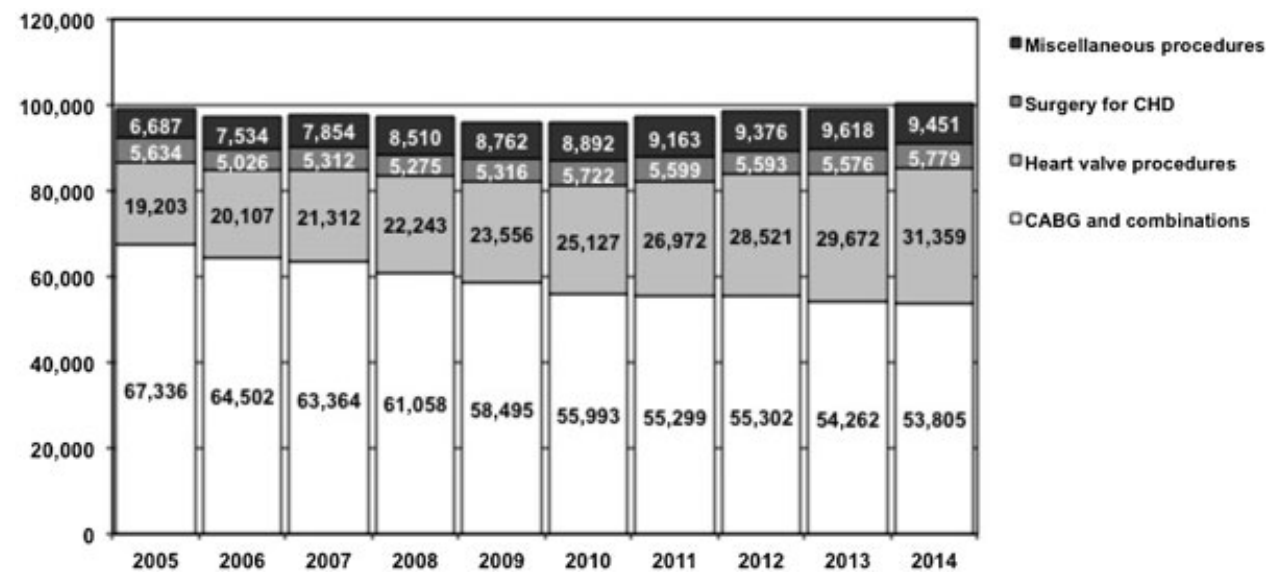

Fig. 1 Cardiac surgery in Germany (2005-2014). (1) Coronary artery bypass grafting (CABG) and combinations: all types of isolated coronary surgery with or without extracorporeal circulation (ECC) and any combined procedure. (2) Heart valve procedures: all types of isolated heart valve surgery; heart valve procedures in combination with aortic surgery are summarized in the miscellaneous group. (3) Congenital heart surgery: all procedures with or without ECC; atrial septal defect repair in adults in combination with CABG or heart valve surgery are summarized in the CABG or heart valve surgery group. (4) Miscellaneous procedures: all other types of procedures with ECC.

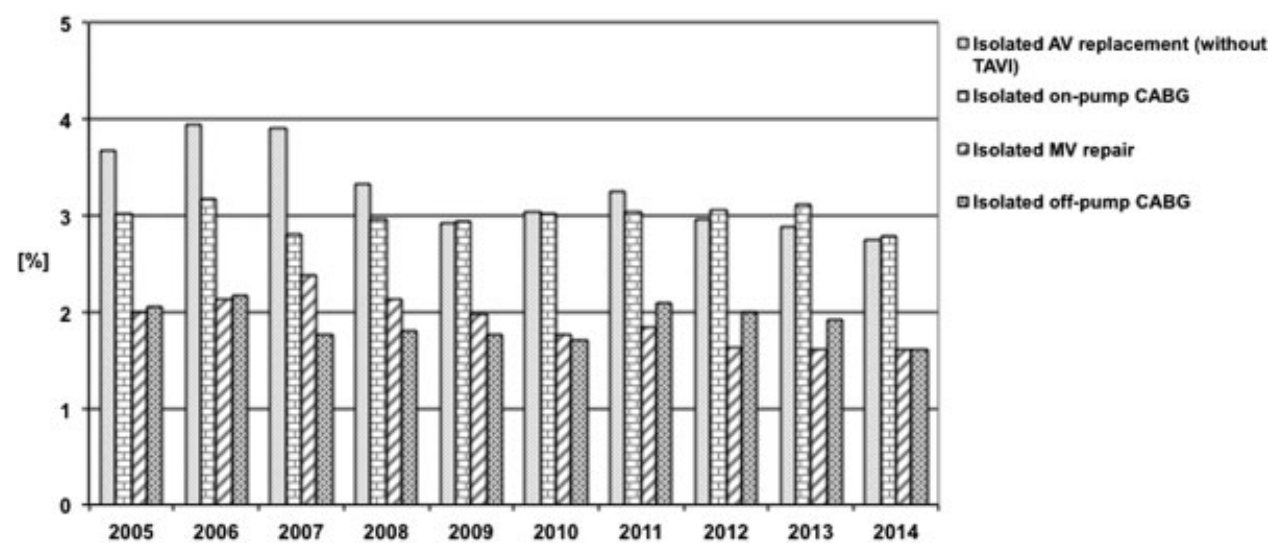

Fig. 2 Unadjusted mortality for selected procedures (2005-2014).

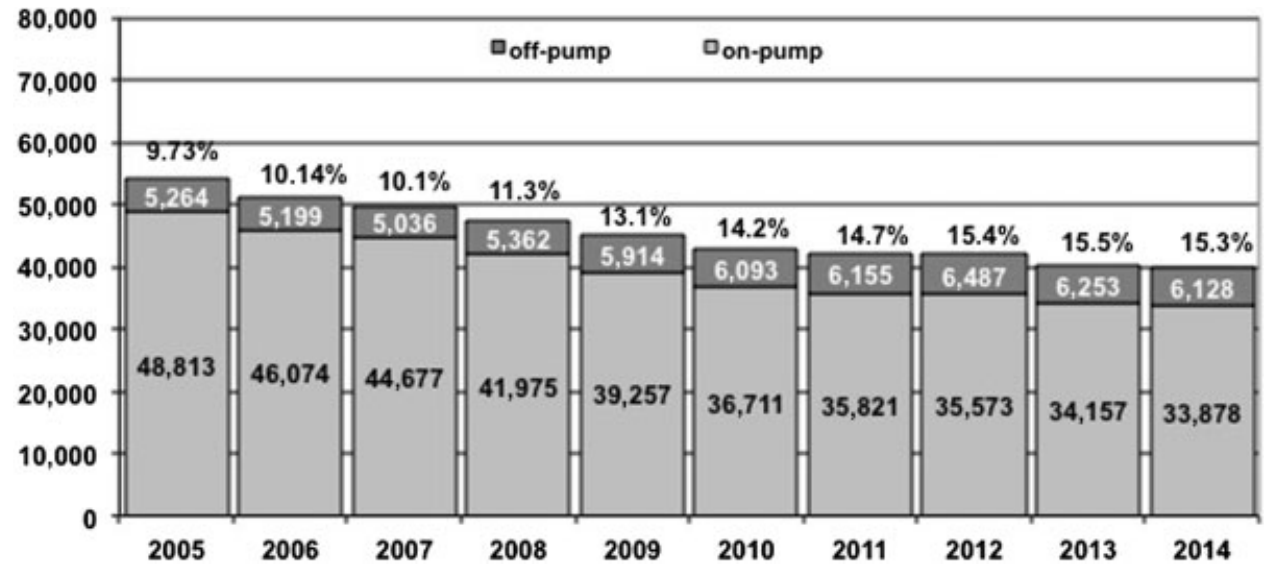

Fig. 3 Isolated coronary artery bypass grafting (CABG) (2005-2014). The quantity of coronary artery bypass procedures declined since the year 2005 while the CABG off-pump procedures reached a nearly constant level of $15.3 \%$. 


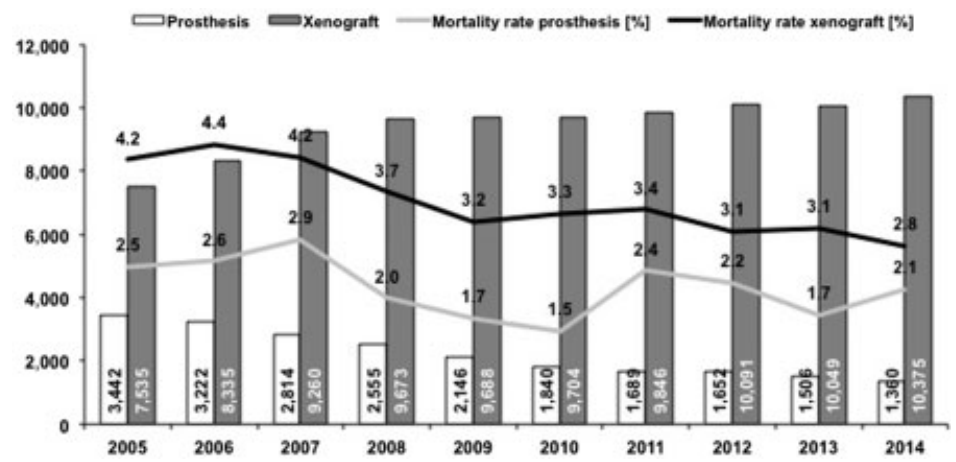

Fig. 4 Isolated aortic valve replacement (2005-2014). The use of xenografts continuously increased till 2008 and reached a stable count since then. AVR implanting mechanical prosthesis decreased over the past 10 years. The observed difference in patients' mortality was probably based on the difference in age pattern and related comorbidities (Ross procedure, homograft implantations, and transcatheter aortic valve implantation are excluded).

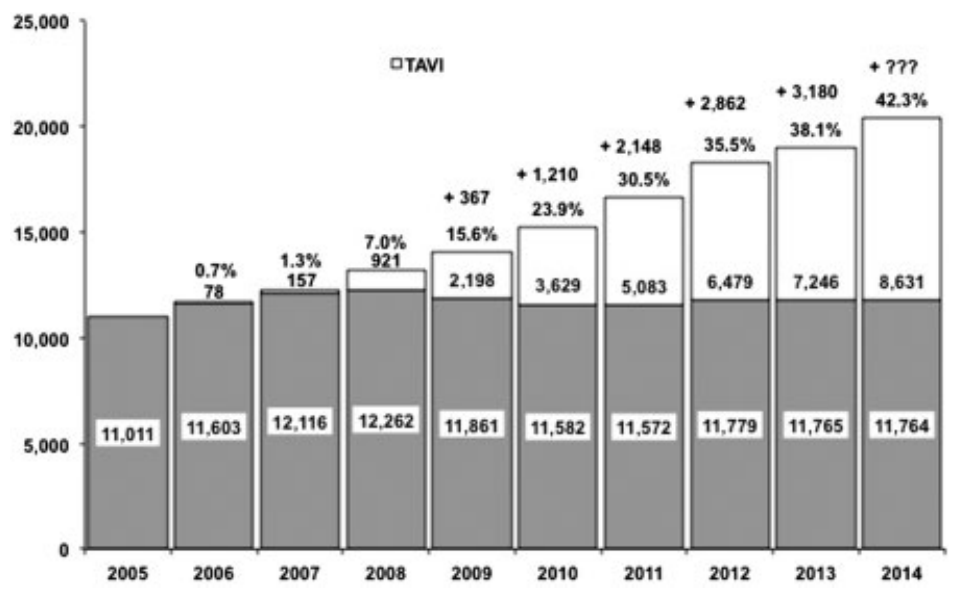

Fig. 5 Isolated AVR and transcatheter aortic valve implantation (TAVI). The figure shows a stable quantity of AVR procedures and a continuous increase of TAVI. In 2014, 8,631 TAVI were reported to the voluntary registry of the GSTCVS; however, this number does not represent all TAVI procedures performed in Germany in 2014. ${ }^{+}$Additional patients with TAVI procedures calculated from the German legal quality assurance program, § 137 SGB V.

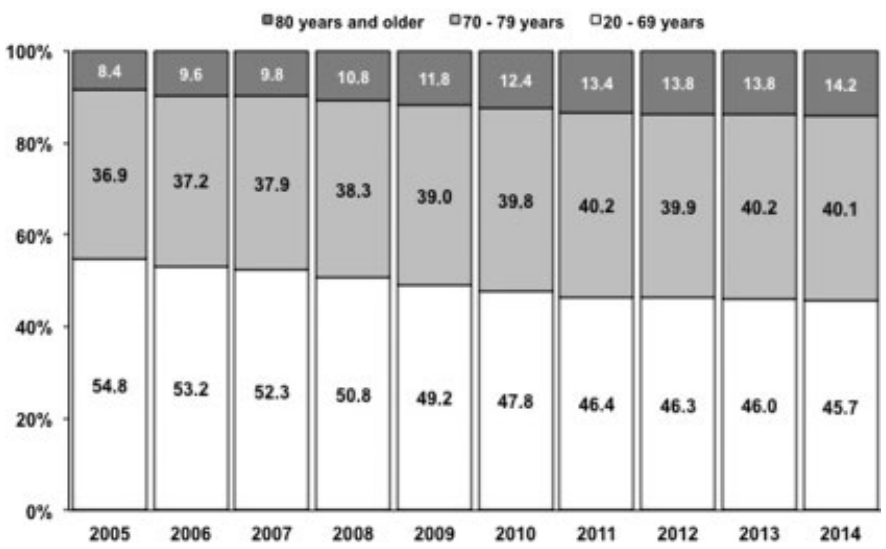

Fig. 6 Age distribution of cardiac procedures (2005-2014). In 2014, 54.3\% of the patients are at least 70 years (patients $<20$ years and ICD/ pacemaker procedures excluded).

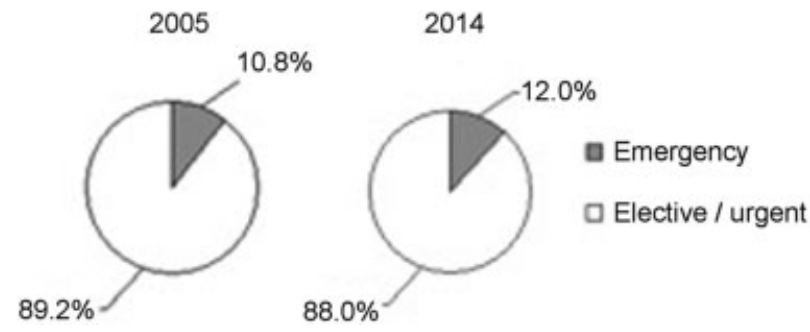

Fig. 7 Procedures timing (2005 vs. 2014). 


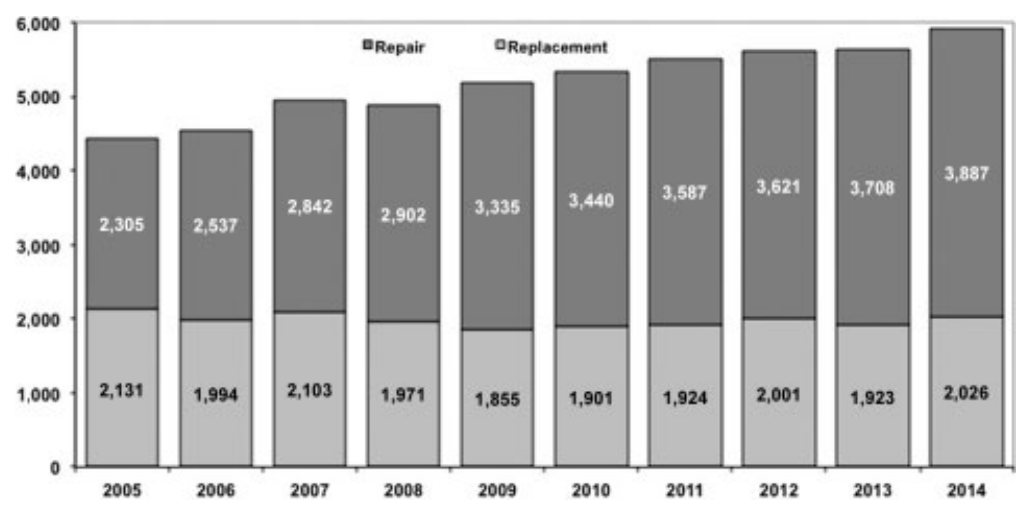

Fig. 8 Isolated mitral valve surgery (2005-2014). In 2014, 65.7\% MV reconstructions and 34.3\% MV replacements were performed. Two decades ago, the repair rate was only $21 \%$.

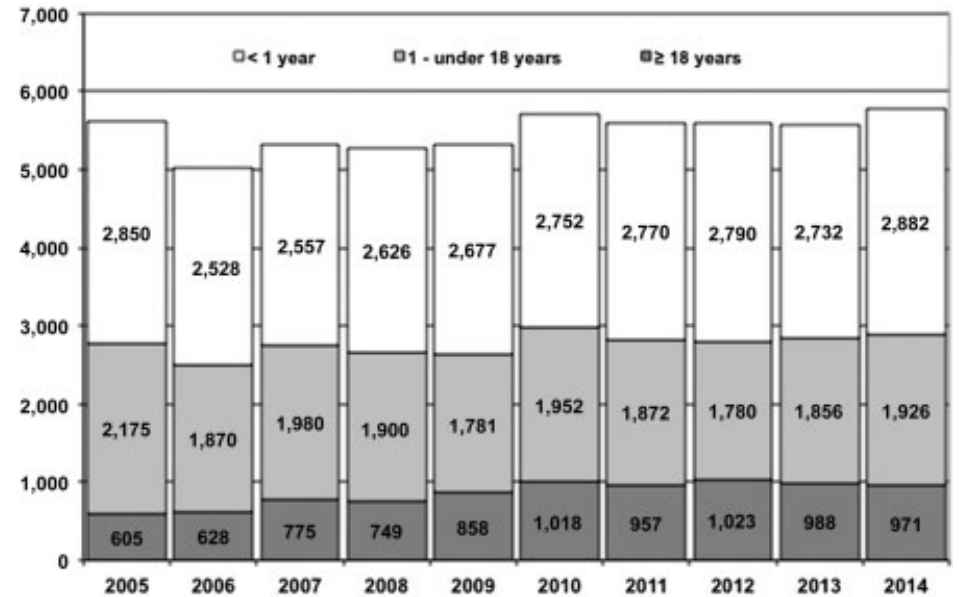

Fig. 9 Age distribution for congenital heart surgery (2005-2014). There are no relevant changes over the past 10 years. However, there may be a bias because not all relevant procedures can be allocated clearly to the congenital heart surgery category for patients older than 18 years (e.g., aortic valve disease).

L/RVAD Implantation

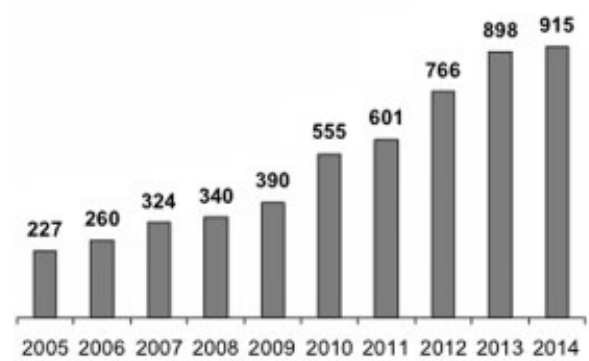

BVAD

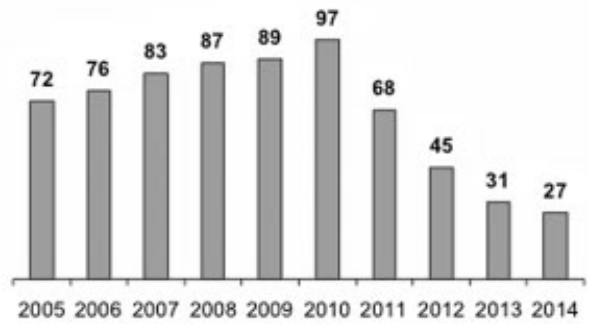

TAH

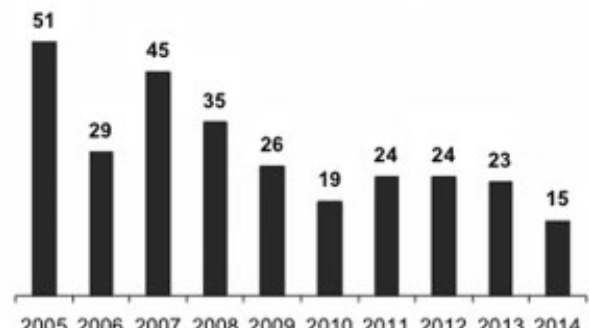

Fig. 10 Development of mechanical circulatory support (2005--2014). Continuous increase of left ventricular assist device (LVAD) implantations. However, the number of implanted paracorporeal biventricular assist devices (BVAD) decreases furthermore and the count of total artificial heart implantations (TAH) dropped down to 15 in 2014. 


\section{References}

1 Kalmar P, Irrgang E. Cardiac surgery in the Federal Republic of Germany during 1989. A report by the German Society for Thoracic and Cardiovascular Surgery. Thorac Cardiovasc Surg 1990;38(3):198-200

2 Kalmar P, Irrgang E. Cardiac surgery in the Federal Republic of Germany during 1990. A report by the German Society for Thoracic and Cardiovascular Surgery. Thorac Cardiovasc Surg 1991;39(3):167-169

3 Kalmar P, Irrgang E. Cardiac surgery in Germany during 1991. A report by the German Society for Thoracic and Cardiovascular Surgery. Thorac Cardiovasc Surg 1992;40(3):163-165

4 Kalmar P, Irrgang E. Cardiac surgery in Germany during 1992. A report by the German Society for Thoracic and Cardiovascular Surgery. Thorac Cardiovasc Surg 1993;41(3):202-204

5 Kalmar P, Irrgang E. Cardiac surgery in Germany during 1993. A report by the German Society for Thoracic and Cardiovascular Surgery. Thorac Cardiovasc Surg 1994;42(3):194-196

6 Kalmar P, Irrgang E. Cardiac surgery in Germany during 1994. A report by the German Society for Thoracic and Cardiovascular Surgery. Thorac Cardiovasc Surg 1995;43(3):181-183

7 Kalmár P, Irrgang E. Cardiac surgery in Germany during 1995. A report by the German Society for Thoracic and Cardiovascular Surgery. Thorac Cardiovasc Surg 1996;44(3):161-164

8 Kalmár P, Irrgang E. Cardiac surgery in Germany during 1996. A report by the German Society for Thoracic and Cardiovascular Surgery. Thorac Cardiovasc Surg 1997;45(3):134-137

9 Kalmár P, Irrgang E. Cardiac surgery in Germany during 1997. A report by the German Society for Thoracic and Cardiovascular Surgery. Thorac Cardiovasc Surg 1998;46(5):307-310

10 Kalmàr P, Irrgang E. Cardiac surgery in Germany during 1998. A report by the German Society for Thoracic and Cardiovascular Surgery. Thorac Cardiovasc Surg 1999;47(4):260-263

11 Kalmár P, Irrgang E. Cardiac surgery in Germany during 1999. Thorac Cardiovasc Surg 2000;48(4):XXVII-XXX

12 Kalmar P, Irrgang E. Cardiac surgery in Germany during 2000. Thorac Cardiovasc Surg 2001;49(5):33-38

13 Kalmár P, Irrgang E. Cardiac surgery in Germany during 2001: a report by the German Society for Thoracic and Cardiovascular Surgery. Thorac Cardiovasc Surg 2002;50(6):30-35

14 Kalmár P, Irrgang E; German Society for Thoracic and Cardiovascular Surgery. Cardiac surgery in Germany during 2002: a report by German Society for Thoracic and Cardiovascular Surgery. Thorac Cardiovasc Surg 2003;51(5):25-29

15 Kalmár P, Irrgang E; German Society for Thoracic and Cardiovascular Surgery. Cardiac surgery in Germany during 2003: a report by the German Society for Thoracic and Cardiovascular Surgery. Thorac Cardiovasc Surg 2004;52(5):312-317

16 Gummert JF, Funkat A, Krian A. Cardiac surgery in Germany during 2004: a report on behalf of the German Society for Thoracic and Cardiovascular Surgery. Thorac Cardiovasc Surg 2005;53(6):391-399

17 Gummert JF, Funkat A, Beckmann A, Hekmat K, Ernst M, Krian A. Cardiac surgery in Germany during 2005: a report on behalf of the German Society for Thoracic and Cardiovascular Surgery. Thorac Cardiovasc Surg 2006;54(5):362-371

18 Gummert JF, Funkat A, Beckmann A, et al. Cardiac surgery in Germany during 2006: a report on behalf of the German Society for Thoracic and Cardiovascular Surgery. Thorac Cardiovasc Surg 2007;55(6):343-350

19 Gummert JF, Funkat A, Beckmann A, et al; German Society for Thoracic and Cardiovascular Surgery. Cardiac surgery in Germany during 2007: a report on behalf of the German Society for Thoracic and Cardiovascular Surgery. Thorac Cardiovasc Surg 2008;56(6): 328-336

20 Gummert JF, Funkat A, Beckmann A, et al. Cardiac surgery in Germany during 2008. A report on behalf of the German Society for Thoracic and Cardiovascular Surgery. Thorac Cardiovasc Surg 2009;57(6):315-323

21 Gummert JF, Funkat A, Beckmann A, et al. Cardiac surgery in Germany during 2009. A report on behalf of the German Society for Thoracic and Cardiovascular Surgery. Thorac Cardiovasc Surg 2010;58(7):379-386

22 Gummert JF, Funkat AK, Beckmann A, et al. Cardiac surgery in Germany during 2010: a report on behalf of the German Society for Thoracic and Cardiovascular Surgery. Thorac Cardiovasc Surg 2011;59(5):259-267

23 Funkat AK, Beckmann A, Lewandowski J, et al. Cardiac surgery in Germany during 2011: a report on behalf of the German Society for Thoracic and Cardiovascular Surgery. Thorac Cardiovasc Surg 2012;60(6):371-382

24 Beckmann A, Funkat AK, Lewandowski J, et al. Cardiac surgery in Germany during 2012: a report on behalf of the German Society for Thoracic and Cardiovascular Surgery. Thorac Cardiovasc Surg 2014;62(1):5-17

25 Funkat A, Beckmann A, Lewandowski J, et al. Cardiac surgery in Germany during 2013: a report on behalf of the German Society for Thoracic and Cardiovascular Surgery. Thorac Cardiovasc Surg 2014;62(5):380-392

26 Gammie JS, Zhao Y, Peterson ED, O’Brien SM, Rankin JS, Griffith BPJ. J. Maxwell Chamberlain Memorial Paper for adult cardiac surgery. Less-invasive mitral valve operations: trends and outcomes from the Society of Thoracic Surgeons Adult Cardiac Surgery Database. Ann Thorac Surg 2010;90(5):1401-1408, 1410.e1, discussion 1408-1410

27 Vahanian A, Alfieri O, Andreotti F, et al; Joint Task Force on the Management of Valvular Heart Disease of the European Society of Cardiology (ESC); European Association for Cardio-Thoracic Surgery (EACTS). Guidelines on the management of valvular heart disease (version 2012). Eur Heart J 2012;33(19):2451-2496

28 G-BA Richtlinie zu minimalinvasiven Herzklappeninterventionen. Available at: https://www.g-ba.de/informationen/beschluesse/2165/

29 Beckmann A, Hamm C, Figulla HR, et al; GARY Executive Board. The German Aortic Valve Registry (GARY): a nationwide registry for patients undergoing invasive therapy for severe aortic valve stenosis. Thorac Cardiovasc Surg 2012;60(5):319-325

30 Hamm CW, Möllmann H, Holzhey D, et al; GARY-Executive Board. The German Aortic Valve Registry (GARY): in-hospital outcome. Eur Heart J 2014;35(24):1588-1598

31 Mohr FW, Holzhey D, Möllmann H, et al; GARY Executive Board. The German Aortic Valve Registry: 1-year results from 13,680 patients with aortic valve disease. Eur J Cardiothorac Surg 2014; 46(5):808-816

32 Lauten A, Figulla HR, Möllmann H, et al; GARY Executive Board. TAVI for low-flow, low-gradient severe aortic stenosis with preserved or reduced ejection fraction: a subgroup analysis from the German Aortic Valve Registry (GARY). EuroIntervention 2014; 10(7):850-859

33 Walther T, Hamm CW, Schuler G, et al; GARY executive board. Perioperative results and complications in 15,964 transcatheter aortic valve implantations from the German Aortic valve RegistrY (GARY). J Am Coll Cardiol 2015. doi: 10.1016/j.jacc.2015.03.034

34 Herbert MA, Prince SL, Williams JL, Magee MJ, Mack MJ. Are unaudited records from an outcomes registry database accurate? Ann Thorac Surg 2004;77(6):1960-1964, discussion 1964-1965

Table V1 has been corrected in the above article as per erratum published online on June 10, 2015. DOI of erratum is: $10.1055 / \mathrm{s}-0035-1556566$. 